\title{
Dysfunctional telomeres in primary cells from Fanconi anemia FANCD2 patients
}

\author{
Ivana Joksic ${ }^{1,2}$, Dragana Vujic ${ }^{3,4}$, Marija Guc-Scekic ${ }^{4,5}$, Andreja Leskovac' ${ }^{1}$, Sandra Petrovic ${ }^{1}$, Maryam Ojani ${ }^{6}$, \\ Juan P Trujillo ${ }^{7}$, Jordi Surralles ${ }^{7}$, Maja Zivkovic ${ }^{1}$, Aleksandra Stankovic ${ }^{1}$, Predrag Slijepcevic ${ }^{6^{*}}$ and Gordana Joksic ${ }^{1^{*}}$
}

\begin{abstract}
Background: Fanconi anemia (FA) is characterized by sensitivity to DNA cross-linking agents, mild cellular, and marked clinical radio sensitivity. In this study we investigated telomeric abnormalities of non-immortalized primary cells (lymphocytes and fibroblasts) derived from FA patients of the FA-D2 complementation group, which provides a more accurate physiological assessment than is possible with transformed cells or animal models.

Results: We analyzed telomere length, telomere dysfunction-induced foci (TIFs), sister chromatid exchanges (SCE), telomere sister chromatid exchanges (T-SCE), apoptosis and expression of shelterin components TRF1 and TRF2. FANCD2 lymphocytes exhibited multiple types of telomeric abnormalities, including premature telomere shortening, increase in telomeric recombination and aberrant telomeric structures ranging from fragile to long-string extended telomeres. The baseline incidence of SCE in FANCD2 lymphocytes was reduced when compared to control, but in response to diepoxybutane (DEB) the 2-fold higher rate of SCE was observed. In contrast, control lymphocytes showed decreased SCE incidence in response to DEB treatment. FANCD2 fibroblasts revealed a high percentage of TIFS, decreased expression of TRF1 and invariable expression of TRF2. The percentage of TIFs inversely correlated with telomere length, emphasizing that telomere shortening is the major reason for the loss of telomere capping function. Upon irradiation, a significant decrease of TIFs was observed at all recovery times. Surprisingly, a considerable percentage of TIF positive cells disappeared at the same time when incidence of $\mathrm{Y}$-H2AX foci was maximal. Both FANCD2 leucocytes and fibroblasts appeared to die spontaneously at higher rate than control. This trend was more evident upon irradiation; the percentage of leucocytes underwent apoptosis was 2.59- fold higher than that in control, while fibroblasts exhibited a 2 - $\mathrm{h}$ delay before entering apoptosis.
\end{abstract}

Conclusion: The results of our study showed that primary cells originating from FA-D2 patients display shorten telomeres, elevated incidence of T-SCEs and high frequency of TIFs. Disappearance of TIFs in early response to irradiation represent distinctive feature of FANCD2 cells that should be examined further.

Keywords: Primary FA cells, Telomere dysfunction, Expression of TRF1 and TRF2

\section{Background}

Located at the ends of chromosomes, telomeres protect chromosomal termini from nucleolytic degradation and erroneous DNA repair; they also prevent activation of DNA damage checkpoints. Human telomeres consist of tandem arrays of a short repetitive DNA sequence (TTAGGG) oriented $5^{\prime}$ to $3^{\prime}$ towards the chromosome and ending in a single-stranded G rich 3' overhang. In human cells, the size of telomeric DNA is genetically regulated, as

\footnotetext{
*Correspondence: predrag.slijepcevic@brunel.ac.uk; gjoksic@vin.bg.ac.rs

${ }^{6}$ Brunel University, West London, United Kingdom

${ }^{1}$ Vinca Institute of Nuclear Sciences, University of Belgrade, Belgrade, Serbia

Full list of author information is available at the end of the article
}

they vary between individuals (e.g., from 10 to $15 \mathrm{~kb}$ in newborns). Telomere length is maintained by dynamic lengthening and shortening. Shortening results from nucleolytic degradation and incomplete DNA replication, whereas lengthening primarily results from telomerase activity, which restores telomeric sequences lost during DNA replication [1]. Telomeric repeats act as binding sites for shelterin: a six-subunit protein complex that protects chromosome ends [2]. Three shelterin subunits, TRF1, TRF2 and POT1, directly recognize TAAGGG repeats. TRF1 and TRF2 bind to double-stranded repeats of the telomere sequence, while POT1 binds single-stranded sites. TRF1 negatively regulates telomere length $[3,4]$ but

\section{Biomed Central}


facilitates the replication of telomeres in S-phase [5]. TRF2 protects chromosome ends by repressing DNA damage induced signaling, non-homologous end joining (NHEJ) and homologous recombination repair (HRR) [6-8]. Telomere dysfunction occurs via the loss of telomere capping function or critical telomere shortening. Both mechanisms lead to the recruitment of DNA damage response (DDR) proteins and formation of telomere dysfunction-induced foci (TIFs) [9-11]. Defects in several DDR factors lead to telomere dysfunction in humans and mice [12]. Fanconi anemia (FA) is characterized by sensitivity to DNA cross-linking agents, mild cellular radio sensitivity and marked clinical radio sensitivity. Dysfunctional telomeres could account for this mild cellular radio sensitivity as a source of endogenous DNA damage. It is already known that FA cells show altered telomere maintenance [13-16], defects in DDR [17] and significant delay in repair kinetics of radiation-induced lesions [18]. We analyzed several telomere maintenance parameters, including telomere length, TIFs and recombination frequencies in the whole genome (sister chromatid exchange $(\mathrm{SCE})$ ) as well as at telomeres (telomere sister chromatid exchange (T-SCE)). In addition, we measured apoptosis and expression of the shelterin components TRF1 and TRF2 following exposure to ionizing radiation and the cross-linking agent diepoxybutane (DEB). The study was performed using non-immortalized primary cells derived from FA patients of the FANCD2 complementation group, providing a more accurate physiological assessment than is possible with transformed cells or animal models.

\section{Results}

Assignment of FA patients to complementation group D2 FA patients were assigned to FA-D2 subtype by immunoblotting. The absence of FANCD2 bands on standard exposure immunoblots suggested that all three patients (1823, 1866 and 1879) belong to complementation group FA-D2 (Figure 1). The over-exposure of the films showed faint FANCD2 bands upon MMC treatment unequivocally indicating that all patients have hypomorphic mutations in FANCD2 as previously described for a larger population of FA-D2 patients [19]. For patient 2093, complementation group analysis was previously performed (Universität Würzburg, Institut für Humangenetik, Germany) and showed that she also belongs to FA-D2 complementation group.

\section{Telomere length analysis in FANCD2 lymphocytes by} quantitative fluorescence in situ hybridization

Representative examples of metaphase spreads hybridized with telomeric PNA probes using Q-FISH and $\mathrm{CO}-\mathrm{FISH}$ protocols are presented in Figure 2. The measurement of telomere length (Figure 3a) revealed that FA-D2 patients exhibited reduced lymphocyte telomere length $(23.50 \pm 8.80)$, relative to age-matched controls $(40.70 \pm 7.36) \quad(\mathrm{P}=0.024)$. The distribution of telomere length (Figure $3 \mathrm{~b}$ ) indicated that, in FANCD2 cells, 5\% of telomeres displayed lengths of 0-10 relative length telomere unit (RTLU), and more than $50 \%$ of all telomeres were only 10-20 RTLU long. In control lymphocytes, the majority of telomeres were 30-50 RTLU long (62.5\%), short telomeres were not present and $15 \%$ of telomeres displayed lengths of 50-80 RTLU. Control lymphocytes displayed typical Poisson distributions for individual chromosome telomere length, whereas the peak of Poisson distribution was shifted leftward in FANCD2 cells. The average percentage of cells displaying fragile telomeres $(18,45 \%)$ and long-extended telomeres $(17,83 \%)$ in FANCD2 lymphocytes are presented in Figure 3c.

\section{Effects of diepoxybutane on the incidence of sister chromatid exchange and telomere sister chromatid exchange in FANCD2 lymphocytes}

The incidence of spontaneous SCE in FANCD2 lymphocytes $(3.87 \pm 0.28)$ was significantly reduced when compared to control $(4.89 \pm 1.16)(\mathrm{P}<0.05)$ (Figure $3 \mathrm{~d})$. In response to DEB, a nearly two-fold increase of SCEs was observed in FANCD2 lymphocytes $(6.68 \pm 1.85)$, relative to the baseline state before treatment $(\mathrm{P}=0.04)$. Appearance of double SCE (dSCE) in response to DEB frequently were observed (Figure $3 \mathrm{e}$ ). In contrast, the same concentration of DEB decreased SCEs in control cells $(4.35 \pm 1.27)(\mathrm{P}=0.034)$.

$\mathrm{CO}-\mathrm{FISH}$ indicated that the incidence of spontaneously occurring T-SCEs in FANCD2 primary lymphocytes $(2.43 \pm 1.96)$ was significantly higher than that of control cells $(P=0.026)$, and further increased upon exposure to DEB $(3.69 \pm 1.68)$. In control cells, the incidence of spontaneously occurring T-SCEs was minimal $(0.32 \pm 0.06)$ and almost unchanged in response to DEB (Figure 3f).

\section{Effects of ionizing radiation on apoptosis of FANCD2 leucocytes and fibroblasts}

As shown in Figure $3 g$ and Figure $3 \mathrm{~h}$ both FANCD2 leucocytes and fibroblasts appeared to die spontaneously at higher rate than control. Upon irradiation the percentage of leucocytes underwent apoptosis was 2.59-fold higher than that in control $(\mathrm{P}=0.0001)$, while in fibroblasts, the mild 2-h delay of entering apoptosis was observed. Both FANCD2 and control fibroblasts extensively enter apoptosis $24 \mathrm{~h}$ after irradiation.

\section{Effects of diepoxybutane and ionizing radiation on percentage of telomere dysfunction-induced foci in FANCD2 fibroblasts}

Representative images of FANCD2 nuclei hybridized with telomeric PNA probes using TIF protocol are presented in 

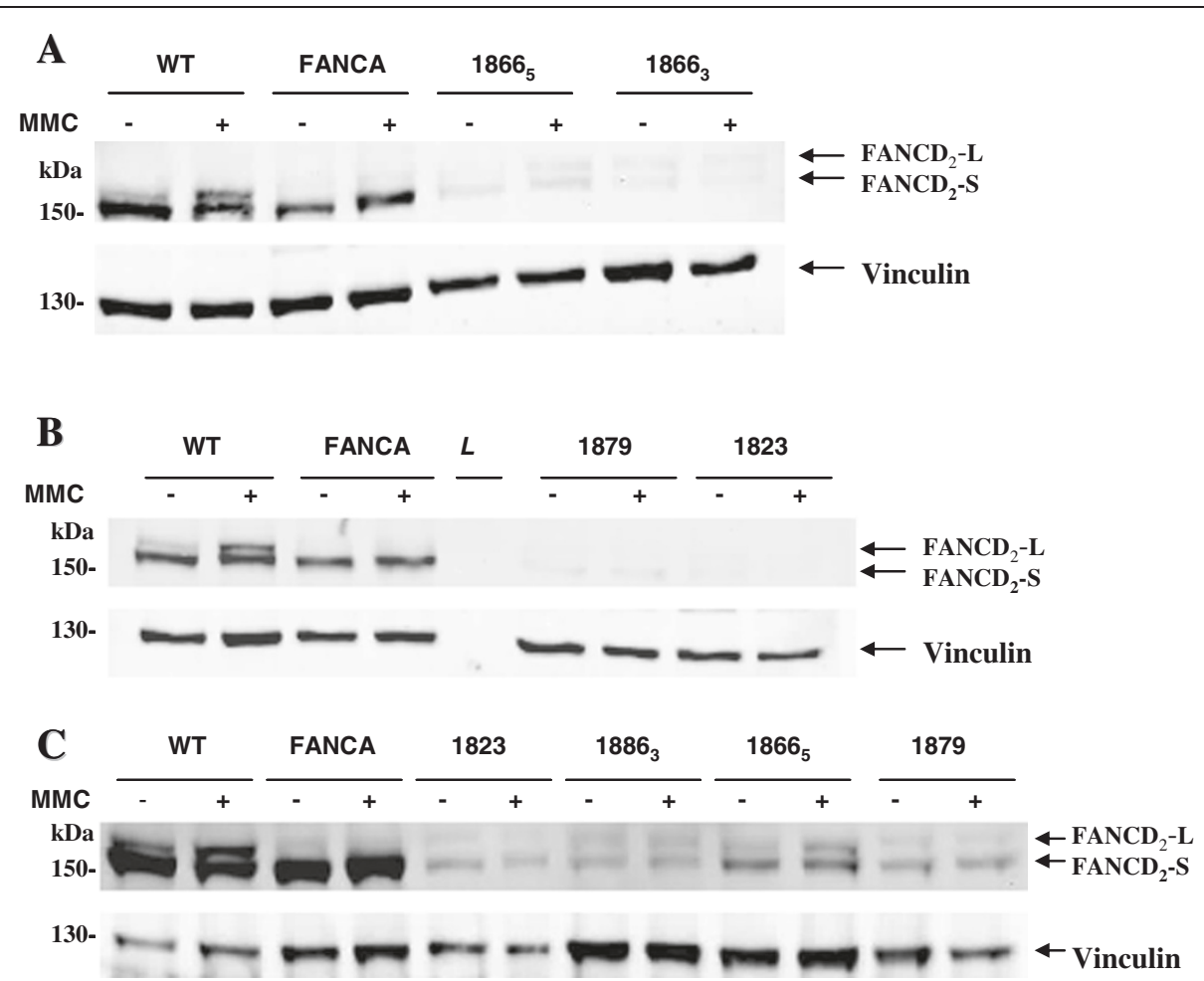

Figure 1 FANCD2 immunoblotting of fibroblast extracts from FA-D2 patients and control cell lines. Exposure of the 1823 , 1866 (2 cell lines from the same patient) and 1879 patient cell lines to 50nM MMC for $24 \mathrm{~h}$ and subsequent analysis by Western blot (loading control Vinculin, L: lane for the Ladder). A Assignment to group FA-D2 of $1866_{3}$ and $1866_{5}$ patient cell lines on the basis of the absence FANCD2 on Western blot. B Assignment to group FA-D2 of 1823 and 1879 patient cell lines on the basis of the absence FANCD2 band on Western blot. C Over-exposure of immunoblots reveals that $1823,1866_{3}, 1866_{5}$ and 1879 patient cell lines show faint but visible FANCD2 bands in response to MMC.

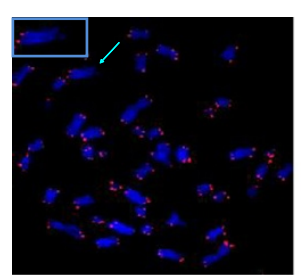

a

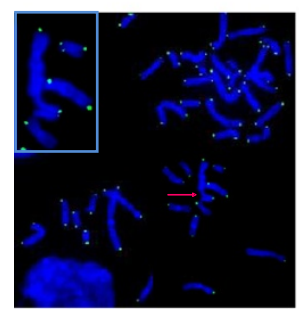

e

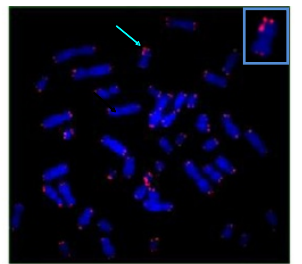

b

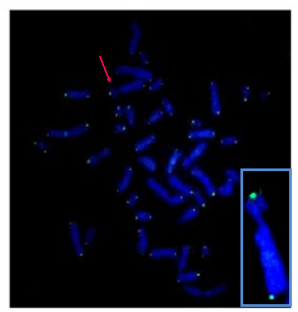

f

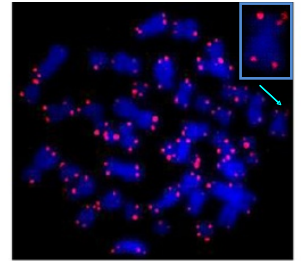

$\mathrm{c}$

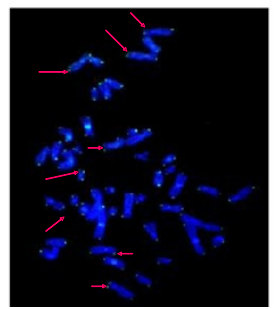

g

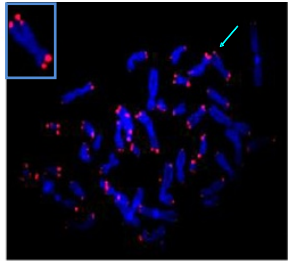

$\mathrm{d}$

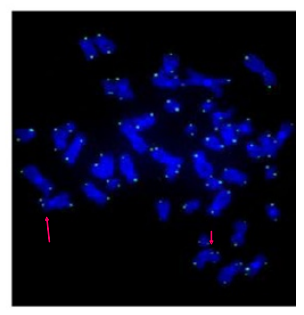

$\mathrm{h}$

Figure 2 Representative images of metaphase spreads hybridized with telomeric PNA probes using Q-FISH (panels a-d) and CO-FISH (panels e-h) protocols. (b-d) Aberrant telomeric structures (blue arrows indicate long strings of fragmented telomeric signals: indicative of fragile telomeres) (e-h) The majority of chromosomes within a metaphase spread showed characteristic CO-FISH hybridization patterns in which telomere signals at opposite ends of the chromosome were in the trans position with respect to the sister chromatids. (a,e and $\mathbf{f})$ Chromatid breakages in metaphase events of FANCD2 cells (arrows indicate no telomeric signals on the breakage points). (g-h) Telomere sister chromatid exchanges (red arrows). 

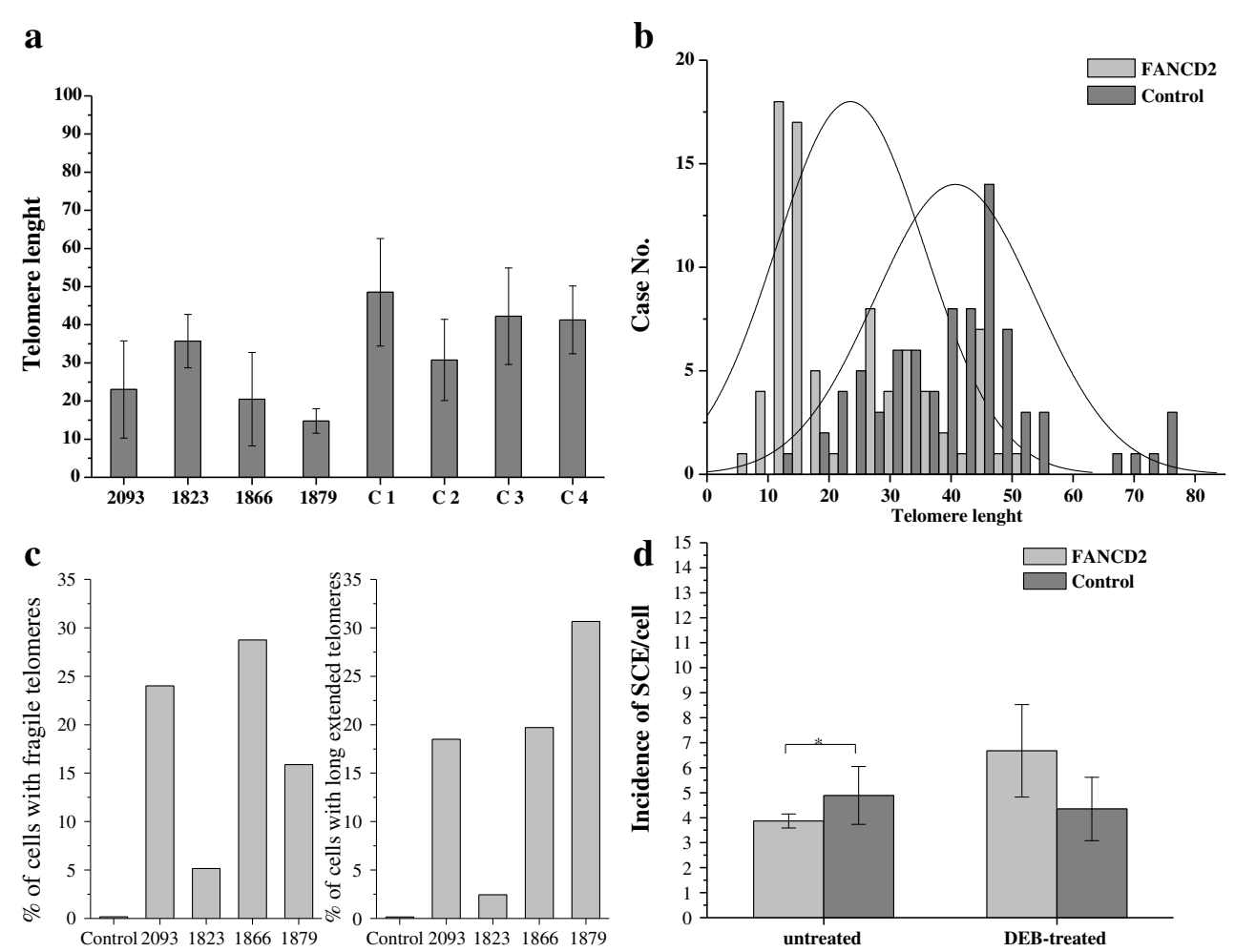

e
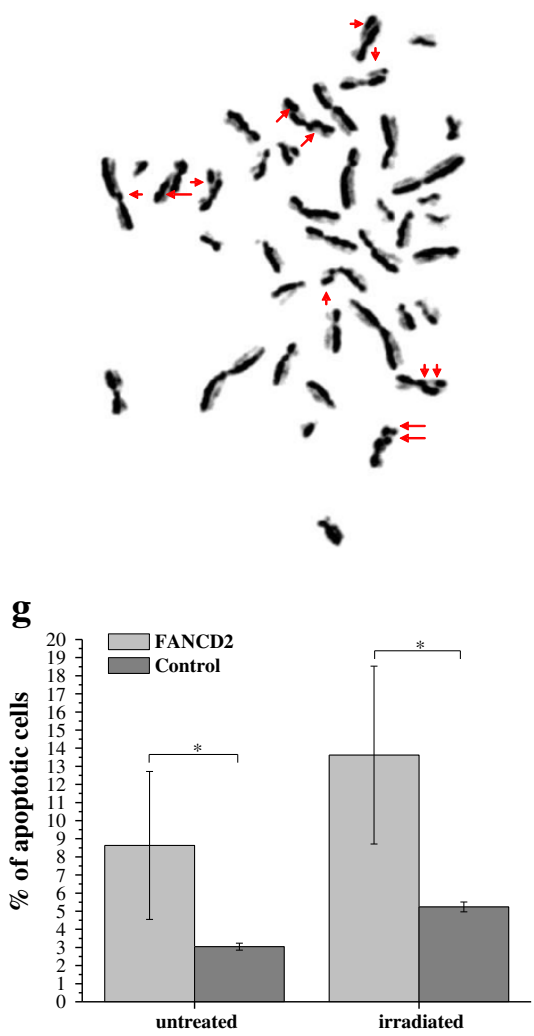

f

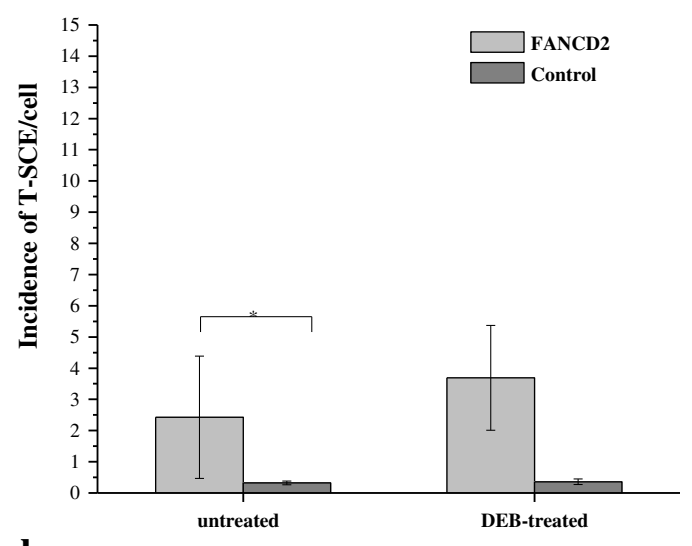

h

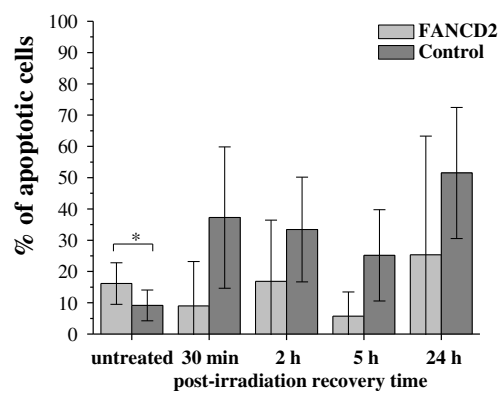

Figure $\mathbf{3}$ (See legend on next page.) 
(See figure on previous page.)

Figure 3 Results of analyses in FANCD2 and control lymphocytes. a Average telomere length (arbitrary units of RTLU \pm SD) measured by QFISH in FANCD2 (1823, 1866, 1879 and 2093) and control (C1-C4) cells. Cells originated from FANCD2 patients display shorter telomeres than the age-matched controls $(p=0.024)$. b The distribution of telomere length indicated that control lymphocytes displayed typical Poisson distributions for individual chromosome telomere length, whereas the peak of Poisson distribution was shifted leftward in FANCD2 lymphocytes. c The percentage of fragile and long extended telomeres: in FANCD2 lymphocytes the percentage of cells displaying fragile telomeres is $18.45 \%$ on the average, wheras percentage of cells displaying long-extended telomeres is $17.83 \%$. d SCE analysis. The incidence of spontaneous SCE in FANCD2 lymphocytes was significantly reduced when compared to control $(p<0.05)$. In response to DEB, almost two-fold increase of SCEs was observed in FANCD2 lymphocytes relative to the baseline state before treatment $(p=0.04)$. Metaphase spread showing DEB treated FANCD2 lymphocytes with 13 SCE and two chromosomes with dSCE . f CO-FISH analysis. The incidence of spontaneously occurring T-SCEs in FANCD2 lymphocytes was significantly higher than that in control cells $(p=0.026)$, and further increased in response to DEB. $\mathbf{g}$ Apoptosis assay showed a significant difference between FANCD2 and control leucocytes in percentage of spontaneously dying cells as well as in percentage of apoptotic cells induced by ionizing radiation. The data are presented as mean \pm SD. $\mathbf{h}$ Apotosis of FANCD2 and control fibroblasts: FANCD2 cells spontaneously die with higher rate than controls and exhibit a mild delay in entering apoptosis. The data are presented as mean \pm SD.

Figure 4. The results of $\gamma-\mathrm{H} 2 \mathrm{AX}$ and TIF analyses in FANCD2 and control fibroblasts are presented in Figure 5. While $\gamma$-H2AX foci were present in both control (5\%) and FANCD2 fibroblasts (13.27\%) enhanced co-localisation of the $\gamma$-H2AX with telomeres was observed only in FANCD2 fibroblasts. As shown in Figure 5a, in average $51.7 \%$ of $\gamma$-H2AX foci positive cells were TIF-positive (displaying more than five TIFs per nucleus). It is worth noting that the level of spontaneously occurring TIFs inversely correlated with telomere length $(P=0.03)$, which emphasizes that shortened telomeres are the underlying cause of their dysfunction.

After DEB treatment, the percentage of TIF-positive FANCD2 cells was significantly reduced $(P=0.026)$ compared to self-state before treatment, whereas in control fibroblasts the percentage of TIF-positive cells slightly increased (Figure 5a).

Upon irradiation, the total number of $\gamma-\mathrm{H} 2 \mathrm{AX}$ foci in FANCD2 fibroblasts was significantly higher at all recovery times, relative to control samples (Figure 5b). Distribution of radiation-induced foci among cells with a recovery time of $30 \mathrm{~min}$, revealed a 2 -fold increase in the number of cells containing more than 30 foci per cell; significantly higher compared to that in control fibroblasts $(\mathrm{P}<0.05)$ (Figure $5 \mathrm{~b})$. Unexpected, at the same recovery time $(30 \mathrm{~min})$ when the incidence of $\gamma$ H2AX foci was maximal significant decrease of TIFs was observed (Figure $5 \mathrm{c}$ ). Afterwards, the percentage of TIF

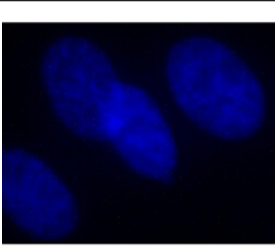

a

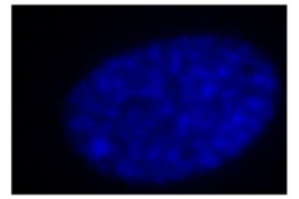

e

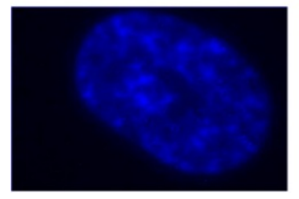

i

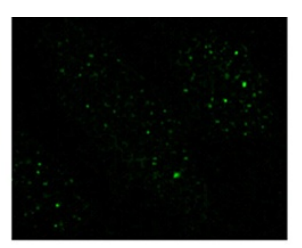

b

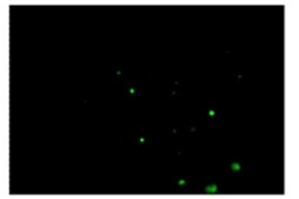

f

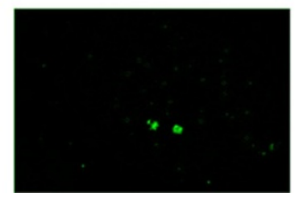

j

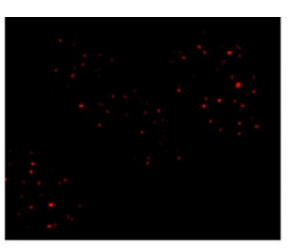

c

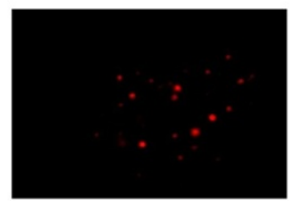

g

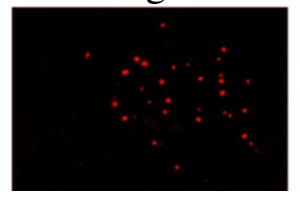

k

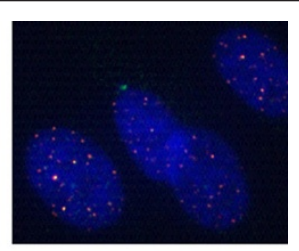

d

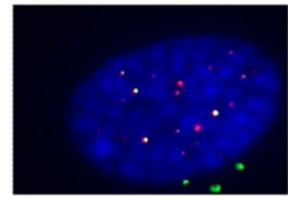

h

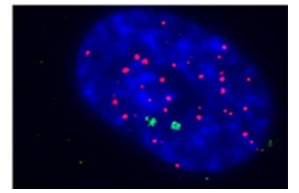

1

Figure 4 Representative images of cell nuclei with or without telomere dysfunction-induced foci (panels a-l). (a-h) Representative examples of FANCD2 nuclei with TIFs. (a, e) DAPI (b, f) $\mathrm{Y}-\mathrm{H} 2 \mathrm{AX},(\mathbf{c}, \mathbf{g})$ Telomeres and $(\mathbf{d}, \mathbf{h})$ Merged. When green and red signals overlap (merge) a yellow pattern is seen, indicating co-localisation of $\mathrm{Y}$-H2AX with telomeres (i-I) Control cell nuclei with distinct $\mathrm{Y}$-H2AX and telomere signals (no TIFs), (i) DAPI, (j) $y-H 2 A X$, (k) Telomeres and (I) Merged. Zeiss-Axioimager A1 microscope equipped with a CCD camera, Axiocam image acquisition software (Imaging Associate) and software package from MetaSystem were used for analysis. 

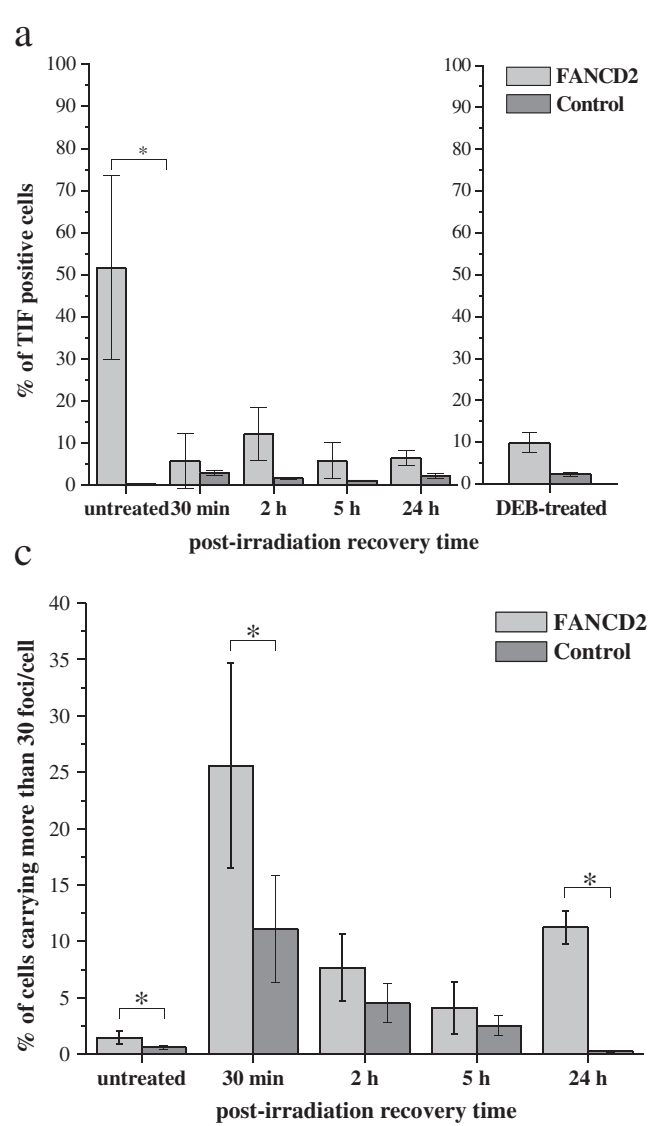

b

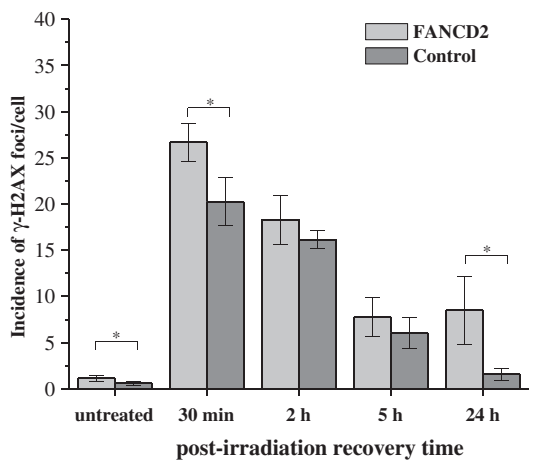

Figure 5 Results of analyses in FANCD2 and control fibroblasts. a Average incidence of radiation-induced $\gamma-\mathrm{H} 2 \mathrm{AX}$ foci per cell (mean \pm SD) in FANCD2 $(n=4)$ and control fibroblasts $(n=6)$. The total number of $\gamma$-H2AX foci in FANCD2 fibroblasts was significantly higher at all recovery times, relative to control samples. b Percentage of FANCD2 cells displaying more than $30 \mathrm{\gamma}-\mathrm{H} 2 \mathrm{AX}$ foci per cell within recovery time of 30 min, revealed a 2-fold increase compared to that in control fibroblasts ( $p<0.05)$. c TIF analysis. In FANCD2 fibroblasts, in average $51.7 \%$ of nuclei were TIF-positive (displaying more than 5 TIFs per nucleus). The level of TIFs inversely correlates with telomere length (P $=0.03$ ). Percentage of TIF positive cells significantly decreased after irradiation. At recovery time of 30 min considerable percentage of TIF positive cells disappeared at the same time when the incidence of $\mathrm{Y}$-H2AX foci was maximal. Afterwards, TIFs appeared again, but in less extent and remained almost the same at all later recovery times. $24 \mathrm{~h}$ after DEB treatment, the percentage of TIF-positive FANCD2 cells also was reduced ( $p=0.026$ ) when compared to a self-state before treatment.

positive cells slightly increased and remained almost the same over the next 2, 5 and $24 \mathrm{~h}$. Percentages of dying FANCD2 fibroblasts were almost the same prior and $2 \mathrm{~h}$ after irradiation (Figure 5d) indicating that cell loss is not the reason for TIFs disappearance. The observations that FANCD2 cells exhibit a mild 2-h delay in entry into apoptosis after irradiation and a 2-fold higher rate of spontaneous apoptosis relative to control signify difficulties in activation of physiological mechanisms of cell death that happened exactly at the time when treated cells did not display TIFs.

\section{Relative expression of TRF1 and TRF2 mRNAs}

Relative to control cells, the relative expression of TRF1 mRNA in FANCD2 fibroblasts was significantly downregulated by a mean factor of 0.31 (S.E. range 0.093 0.610, 95\%CI 0.088-0.697, $\mathrm{P}=0.000$ ) (Figure 6). TRF2 gene expression was similar in both FANCD2 and control cells (mean factor 1.178, S.E. range 0.814 - 1.71, 95\% CI $0.771-1.801, P=0.7)$. The TRF1:TRF2 ratio was close to $1(\mathrm{P}=0.7)$ in control cells. We did not detect significant differences in TRF1 or TRF2 gene expression between FANCD2 and control cells following irradiation or treatment with DEB.

\section{Discussion}

This study provides evidence that primary cells originating from FA-D2 patients display shorter telomeres than their age-matched control counterparts, high frequency of dysfunctional telomeres, and broad spectrum of aberrant telomeric structures ranging from fragile to longstring extended telomeres. Furthermore, telomeres in FANCD2 primary cells display extra heterogeneous telomere lengths of individual chromosomes and elevated 


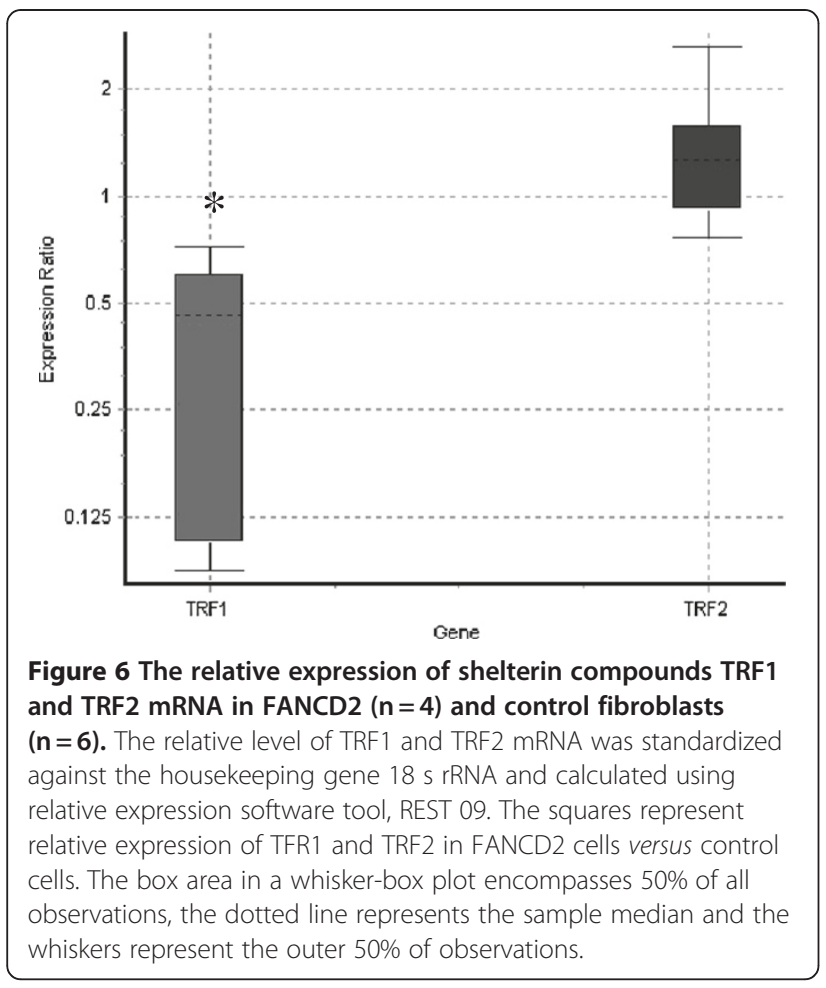

incidence of T-SCEs. The recent work of Ball et al. [13], Callen et al. [14] and Leteurtre et al. [20] have demonstrated the presence of shorter telomeres in FA patients (mostly belonging to the complementation group FA-A) than their age-matched controls, which is consistent with our findings in FA-D2 patients. Herman et al. [21] proposed that average telomere length is not the only determinant of telomere function, emphasizing that critically short individual telomeres can trigger cellular responses to a loss of telomere function. The heterogeneity of telomere length that was present in our FA-D2 patients was expressed as SD of telomere length; our correlative analysis supports their hypothesis that critically short telomeres trigger a DDR response that reduces telomere capping function. Metaphase events presented in Figure 2(b-d), illustrate a hybridization pattern with aberrant telomeric structures ranging from fragmented telomeric signals to an extended strand of telomeric DNA; these patterns are indicative of telomere dysfunction. The findings of this study support those reported in several prior studies [22-26] with embryonic stem cells lacking TRF1. In this study, all FA-D2 patients exhibited decreased TRF1 expression, which correlates to telomere defects. Because TRF1 has been described as a negative regulator of telomere length [27], reduced TRF1 expression is thought to induce telomere elongation; however, this did not occur. In contrast to tumor cells $[28,29]$, down-regulation of TRF1 in FANCD2 cells is probably linked to the functional role of TFR1 in regulating cell cycle progression. In response to DNA damage, ATM beside histone H2AX, phosphorylates TRF1 to inhibit its mitotic function and prevent abortive mitosis. Given that FANCD2 cells exhibit a 2-fold increase in the number of cells displaying more than $30 \gamma \mathrm{H} 2 \mathrm{AX}$ foci per cell after irradiation, down-regulation of TRF1 may be a consequence of its degradation due to the failure of cells to progress into the cell cycle normally, as is the case with control cells. Down-regulation of TRF1 may also provide cells to increase their proliferative potential.

By employing the CO-FISH technique, our study demonstrates a two-fold enhancement of T-SCEs in FANCD2 cells, relative to control cells. This may result from an attempt to replenish lost telomeric sequences by homologous recombination between sister telomeres. While there is currently no direct evidence that T-SCEs modify telomere length, the recombinogenic behavior of telomeres in FANCD2-deficient cells could contribute to heterogeneity in telomere length, which in turn activates DDR. Recent work by Lyakhovich et al. [30] with immortalized FANCD2 fibroblasts has shown that extrachromosomal circular telomeric structures in FANCD2-deficient cells are not linked to telomeric recombination between sister chromatids. In our study, FISH analysis of primary FANCD2 cells did not reveal extrachromosomal telomeric signals; in contrast, fragile telomeres and split or extended telomeric signals were frequently observed. Recent work of Polanska et al. [31], have shown that chromatin associated protein (HMGB1) also maintain telomere integrity. The most interesting finding in their study is that $\mathrm{HMGB}^{-1-}$ cells exhibit heterogeneous telomere lengths of individual chromosomes and highly extended telomeres, as we found in lymphocytes of our FANCD2 patients.

Considering the role of TRF2 in telomere maintenance, many studies with mouse models have revealed that T-SCE are repressed by TRF2 [32]. Expression of TRF2 in either FA cells or control cells was nearly identical, indicating that an increase of T-SCE could be attributed to some other proteins; examples of which include those that regulate histone heterochromatin density in telomeric and sub-telomeric regions and influence chromatin remodeling, as suggested by Schoeftner and Blasco [33], and Polanska et al. [31].

The novel finding of our study is that a remarkably high percentage of FANCD2 cells had dysfunctional telomeres that were mostly caused by telomere shortening collectively with extensive telomere length heterogeneity of individual chromosomes (i.e., 1866 cells displayed 74\% of TIF positive cells). Although elevated incidence of $\gamma \mathrm{H} 2 \mathrm{AX}$ foci in FANCD2 cells vs. control was observed at all recovery times after irradiation, a significant decrease of TIFs was determined at the same post-irradiation times. Particularly unexpected was a considerable disappearance of TIF 
positive cells $30 \mathrm{~min}$ after irradiation, when the incidence of $\gamma \mathrm{H} 2 \mathrm{AX}$ foci was maximal (Figure 5a). Afterwards, TIFs appeared again, but in less extent and remained almost the same at all later recovery times. When we look at the apoptosis profile of FANCD2, it is evident that percentage of dying cells was almost the same up to $2 \mathrm{~h}$ after irradiation (compared to a baseline state), indicating that cell loss is not the reason for TIFs disappearance. The work of Kaul et al. [34] have shown that normal human cells can tolerate small number of TIFs, so called "intermediate-state" telomeres. We hypothesize that repair-deficient FANCD2 cells could possibly tolerate bigger number of intermediate-state telomeres. However, upon irradiation FANCD2 cells display fast and marked reduction of TIFs, which is likely due to a recruitment of $\gamma \mathrm{H} 2 \mathrm{AX}$ away from telomeres to newly formed DSBs. Irradiated FANCD2 fibroblasts exhibit mild delay of $2 \mathrm{~h}$ in ingoing apoptosis relative to control, signifying difficulties in activation of physiological mechanisms of cell death. Accelerated telomere shortening in radiosensitive cell lines has been reported in several prior publications $[35,36]$. Collectively, the data presented by our study suggests that uncapping of telomeres results from an increase in telomere recombination, in addition to accelerated telomere shortening; this is consistent with general enhancements in the activity of HR. For example, 1823 cells possess telomeres of nearly normal length and display no increase in HR activity; therefore, we conclude that only cells with sufficiently long telomeres bypass HR to replenish telomeric repeats. In FANCA cells, telomere dysfunction-induced foci were found in less than $10 \%$ of cells. FANCA also exhibit shorter telomeres than controls, but in contrast to FANCD2 exhibit fast progression, no delay in cell cycle and display very few TIFs in base-line state, and in response to irradiation behave in a similar manner as controls (unpublished data).

In response to cross-linking agents, FANCD2 lymphocytes exhibited a 2-fold increase in the frequency of SCE when compared to control cells. In control lymphocytes, progression of SCE in response to DEB was suppressed. To the best of our knowledge, this is the first report that describes a role of the cross-linking agent DEB in suppressing the occurrence of SCE in normal cells and significantly enhancing it in FA cells. Particularly frequent double SCE were observed, as illustrated in Figure 3f. Increased SCE incidence upon DEB treatment suggests that replication fork collapse may occur because of the inability of cells to repair replication-blocking lesions and initiation of homologous recombination. According to findings in our study incidence of SCE induced by DEB can be used as an adjunct diagnostic test for FA cellular phenotype. High-rates of homologous recombination in human FA cells first were observed by Thyagarajan and Campbell [37], who discovered that recombination levels in Fanconi anemia cells were nearly 100-fold higher than in control cells. The authors proposed that inappropriate increase in the amount of homologous recombination might contribute to genomic instability and cancer susceptibility that characterized FA. Rouet et al. [38] found that HR processes operate on DSBs, which can further rationalize our finding that enhanced HR activity was present in treated FA-D2 cells [38]. Work of Yamamoto et al. [39] have shown that sensitivity of FANCD2 cells to cross-linking agents and ionizing radiation is mainly due to premature termination of repair during DNA synthesis. Nakanishi and colleagues [40] provide evidence that cells derived from FANCA, FANCG and FANCD2 patients are defective, to a mild degree in HR. Smogorzewska et al. [41] verify that siRNA mediated depletion of FANCI and FANCD2 resulted in reduced HR activities. Indeed, the results of our study revealed reduced rate of SCE in FANCD2 cells and higher rate of spontaneously dying cells versus control, which is in accordance to previously mentioned findings.

The mechanism by which telomere dysfunction is initiated remains unresolved. Broccoli et al. [42] postulated that dysfunctional telomeres may fall below a threshold of telomere-associated proteins, such as TRF1 and TRF2, thereby impairing the formation of the telomere loop structure; however, subsequent studies, including our study, determined that this effect is not connected to these important shelterin components. Morish and Greider [43] reported that mammalian cells utilize several different non-telomerase mechanisms for telomere maintenance, which include recombination initiated by short telomeres; the results of our study support this hypothesis. The work of Fan and colleagues [44] have shown that depletion of FANCD2 is associated with decrease in recombination between telomeres. In our study the opposite results were obtained, which can be explained in part by the fact that truncated FANCD2 protein in primary FA-D2 cells is still competent to function in HR. Telomere findings in our study confirm that FANCD2 cells maintain telomeres trough homologous recombination displaying a more heterogeneous length and increased chromosomal instability including TIFs. Interestingly no fusions were observed in either lymphocytes or fibroblasts indicating that telomeres are not critically shorten to be fused. This observation is in line with work of Cesare and colleagues who postulated that not all uncapped telomeres are equal, pointing out that some cells as cancerous cells posses TIF positive telomeres that are not involved in fusions [45]. The similar distinctiveness of telomeres in primary cells originated from FA-D2 patients are observed in our study.

Taken together, our study revealed that high percentage of TIFs that characterized FANCD2 primary cells is most likely induced by critically shortened telomeres. In addition, fragile and long-string extended telomeres along with disappearance of TIFs in early response to irradiation are distinctive features of FANCD2 primary 
cells. Genomic instability resulting from telomere dysfunction is a plausible explanation for the increased cancer susceptibility and clinical hypersensitivity of all FA patients, not only of the FA-A complementation group.

\section{Methods}

\section{Patients}

The patients included in this study were four female children with Fanconi anemia belonging to complementation group FA-D2. The patients were $8 \pm 5$ years of age. Cellular FA phenotype was diagnosed with classic chromosomal breakage DEB tests of peripheral blood lymphocytes, as described by Auerbach et al. [46]. Patients who displayed DEB-positive lymphocyte phenotypes underwent skin biopsies to assess FA fibroblast phenotype and to determine genetic subtype. Healthy age-matched volunteers served as control groups (12 subjects as controls for SCE analysis, and 4 subjects as controls for telomere length analysis). All methods were approved by the Ethical Committee of the Mother and Child Health Care Institute of Serbia, and the subjects' parents signed informed consent forms.

\section{Cell culture}

\section{Lymphocytes}

Peripheral blood was collected from all subjects and deposited into heparinized vacutainer tubes. Lymphocytes were cultured in PBmax karyotyping medium (Invitrogen-Gibco, Paisley, UK). Adequate numbers of cell cultures per subject were established, enabling SCE analysis, T-SCE analysis and telomere length analysis via quantitative FISH (Q-FISH) in metaphase cells.

\section{Fibroblasts}

Cells were grown in DMEM (Invitrogen-Gibco, Paisley, UK) supplemented with 10\% fetal bovine serum (Invitrogen-Gibco, Paisley, UK) under standard tissue culture conditions: $37^{\circ} \mathrm{C}$ in $10 \% \mathrm{CO}_{2}$. Cells were harvested in duplicate and propagated to $80-90 \%$ confluence, at which point cells were subcultured. A $1.0 \mathrm{~mL}$ cell suspension was prepared at a density of 50,000 cells for each sample, and the cells were seeded onto polylysine-coated glass slides (Sigma Chemical Co., St Louis, MO) and incubated overnight in atmosphere-controlled chamber containing $10 \% \mathrm{CO}_{2}$. As a control, primary cells from skin biopsies of six healthy subjects undergoing plastic surgery were also used.

\section{Irradiation}

Irradiation was performed with a ${ }^{60} \mathrm{Co} \gamma$-ray source (a 2.0 Gy dose at $0.45 \mathrm{~Gy} / \mathrm{min}$ ). Fibroblasts were irradiated on polyprep slides and then returned to the tissue culture incubator and examined at various recovery times (30 min, $2 \mathrm{~h}, 5 \mathrm{~h}$ and $24 \mathrm{~h}$ ).

\section{Diepoxybutane treatment}

Cells were treated with DEB at final concentration of $0.1 \mu \mathrm{g} / \mathrm{mL}$ or $0.01 \mu \mathrm{g} / \mathrm{mL}$ for lymphocytes and fibroblasts, respectively. Fibroblasts were exposed to DEB for $24 \mathrm{~h}$, whereas lymphocytes were exposed for $72 \mathrm{~h}$.

\section{Western blot analysis for the subtyping of FA patients}

Exposure of the patients and controls cell lines to $50 \mathrm{nM}$ mitomycin $\mathrm{C}(\mathrm{MMC})$ for $24 \mathrm{~h}$ and subsequently analysis by FANCD2 western blot was performed as previously described by Casado et al. [47] and Castella et al. [48]. Anti-FANCA antibodies (gift of the Fanconi Anemia Research Fund, dilution 1:500) and anti-FANCD2 antibodies (Santa Cruz Biotechnology, Santa Cruz, California, USA) (dilution 1:2500) were used. Assignment of group FA-D2 was done on the basis of the absence FANCD2 on Western blots usingWestern Breeze Immunodetection Kit (Invitrogen, Grand Island, NY, USA). The over-exposure of films by the chemiluminescence technique to identify residual FANCD2 protein levels was performed as described by Kalb et al. [19].

\section{Quantitative fluorescence in situ hybridization}

For Q-FISH, cells were harvested under standard conditions. During the final $3 \mathrm{~h}$ of incubation, $0.1 \mu \mathrm{g} / \mathrm{mL}$ of colchicine (Sigma Chemical Co., St Louis, MO) was added to cell cultures. Subsequently, a hypotonic solution $(5.6 \mathrm{~g} / \mathrm{l}$ of $\mathrm{KCl})$ was added, followed by three consecutive fixations with 3:1 methanol/acetic acid; thereafter, slides were prepared. Q-FISH was performed as described by Slijepcevic [49]. Briefly, after appropriate wash steps, the slides were hybridized with the Cy-3 labeled telomeric PNA probe (CCCTAA) 3' and left in a dark humidified chamber for $2 \mathrm{~h}$. The slides were then washed in $70 \%$ formamide and stained with 4', 6'-diamidino-2-phenylindole (DAPI)-containing mounting medium (Vector Laboratories, UK). Chromosomal analysis was performed with a ZiessAxioplan2 microscope equipped with a CCD camera, Axiocam image acquisition software (Imaging Associate) and software package from MetaSystem. Measurements were reported as arbitrary relative telomere length units (RTLU), which are defined as the ratio of signal intensity between telomeres and a centromere chromosome 2 reference signal.

\section{Sister chromatid exchange analysis}

Cells intended for SCE analysis were cultured in duplicate and allowed to undergo two rounds of replication in the presence of 5-bromo-2-deoxyuridine (BrdU) (Sigma Chemical Co., St Louis, MO) to achieve differentiation of sister chromatids. BrdU was added one hour after culture initiation and cells were harvested at $72 \mathrm{~h}$, the final three hours of which were conducted in the presence of colchicine (final concentration $0.1 \mu \mathrm{g} / \mathrm{mL}$ ). One of 
these cultures was used to analyze spontaneously occurring SCE, while a second was treated with DEB one hour after culture initiation, and continued for as long as $72 \mathrm{~h}$. After conventional hypotonic treatment, cells were fixed three times in 3:1 methanol: acetic acid. Afterward, the pellet was spread onto glass microscope slides. Differential staining of sister chromatids was performed via standard fluorescence-plus-Giemsa (FPG) technique [50]. A total of 30 complete second-division metaphase cells were analyzed per sample with a Ziess-Axioplan2 microscope.

\section{Chromosome orientation fluorescence in situ hybridization}

Parallel cultures were used to conduct telomere T-SCE analysis. The chromosome orientation fluorescence in situ hybridization (CO-FISH) protocol of Bailey et al. [51] was performed with some modification for lymphocytes: $48 \mathrm{~h}$ after the start of culture, $30 \mu \mathrm{M}$ BrdU and $10 \mu \mathrm{M}$ bromodeoxycytidine (BrdC) (Sigma Chemical Co., St Louis, MO) were added and cells were allowed to progress through $S$ phase and into the first mitosis the following day. Colchicine was added to a final concentration of $0.1 \mu \mathrm{g} / \mathrm{mL}$ during the last $3 \mathrm{~h}$ of culture. Preparation of cultures was performed according to the standard protocol (hypotonic treatment and three consecutive fixations in 3:1 methanol: acetic acid). Cell pellets were spread onto glass microscope slides using cytogenetic techniques. Newly synthesized DNA strands were degraded by treating fixed cells on glass microscope slides with $0.5 \mu \mathrm{g} / \mathrm{mL}$ Hoechst 33258 for 15 min (Sigma Chemical Co., St Louis, MO), 313 nm light for $30 \mathrm{~min}$ and $3 \mathrm{U} / \mu \mathrm{L}$ Exo III (Promega, Madison, WI) at room temperature for $10 \mathrm{~min}$. Slides were washed in PBS and subsequently dehydrated in a series of ethanol washes. Tel Cy3-dUTP $(20 \mu \mathrm{L})$, a telomere PNA probe (Panagene, Korea) was placed onto each slide, covered with glass coverslips and hybridized on a hot plate for $5 \mathrm{~min}$ at $80^{\circ} \mathrm{C}$. After hybridization, slides were maintained in the dark in a wet compartment for $2 \mathrm{~h}$. Afterwards, the slides were washed three times in $70 \%$ formamide $/ 2 x$ SSC for $15 \mathrm{~min}$, and then washed three times in PBS for $5 \mathrm{~min}$. Samples were then dehydrated in ice-cold ethanol, mounted in DAPI (Vectashield), covered with coverslips and sealed. At least 20 metaphase events from each patient were quantified by CO-FISH. Each metaphase event was analyzed with Axiocam image acquisition software (Imaging Associate) and a software package from MetaSystem. The data were subsequently analyzed by quantifying descriptive statistics (mean values, SD and SE).

\section{TIF assay}

For the TIF assay, cells were co-stained with $\gamma \mathrm{H} 2 \mathrm{AX}$ and telomere in situ hybridization using a telomere-specific peptide nucleic acid probe. Polylysine-coated slides on which cells were grown were rinsed in PBS and fixed in
$4 \%$ formaldehyde for $15 \mathrm{~min}$. Cells were permeabilized in $0.2 \%(\mathrm{v} / \mathrm{v})$ Triton-X in distilled water at $4.0^{\circ} \mathrm{C}$ for $10 \mathrm{~min}$ and blocked with $0.5 \%(\mathrm{w} / \mathrm{v})$ bovine serum albumin (BSA) in PBS for 30 min. Anti-phospho- histone H2AX (Ser139) (Millipore, USA) was diluted 1:500 with 0.5\% BSA; $100 \mu \mathrm{L}$ of the resulting solution was added to each slide and incubated for $1 \mathrm{~h}$ in a humidified container. After three washes in sterile tris-buffered saline containing tween-20 (TBS-T, pH7.4; $0.15 \mathrm{M} \mathrm{NaCl}, 0.268 \mathrm{mM} \mathrm{KCl}, 0.025 \mathrm{M}$ tris-base, $500 \mu \mathrm{L} / 1 \mathrm{l}$ tween-20) for $3 \mathrm{~min}$, slides were incubated with $100 \mu \mathrm{L}$ of anti-goat secondary antibody conjugated to fluorescein isothiocyanate (FITC) and washed as described above. The slides were then placed in $4 \%$ formaldehyde for $20 \mathrm{~min}$ to facilitate cross-fixing and antibody preservation. The following step involved hybridization to a telomeric PNA probe, which was performed as described above with the exception that TBS-T was used for washing instead of PBS. A large number of cells (100) were analyzed for each sample. TIFs were measured before irradiation (baseline state) and at different times after exposure to ionizing radiation ( $30 \mathrm{~min}, 2 \mathrm{~h}, 5 \mathrm{~h}$ and $24 \mathrm{~h}$ ) and $24 \mathrm{~h}$ after DEB treatment.

\section{Apoptosis assay}

Blood cells: For apoptosis assay, aliquots of $0.5 \mathrm{ml}$ peripheral blood from each subject were cultured in duplicate. One of these cultures was used for analysis of spontaneous apoptosis, while the second culture was set up by using the irradiated whole blood. Blood cells were incubated in medium RPMI-1640 supplemented with $15 \%$ of calf serum without phytohemaglutinin (PHA) in $\mathrm{CO}_{2}$ incubator for $24 \mathrm{~h}$. After incubation, cells were gently washed with physiological saline $(0.9 \% \mathrm{NaCl})$ at $37^{\circ} \mathrm{C}$, fixed in methanol: acetic acid (3:1) and subsequently fixed in 96\% ethanol.

Fibroblasts: At each time point after irradiation and/or DEB treatment cells were detached from the flasks surface with 0.025 M trypsin-EDTA (Gibco, Invitrogen Ltd., Paisley, UK), washed with pre-warmed PBS (Bioatlas, Tartu, Estonia) at $37^{\circ} \mathrm{C}$ and fixed in $96 \%$ ethanol. Apoptosis was assessed by flow cytometric (Becton Dickinson, Heidelberg, Germany) identification of cells displaying apoptosis associated DNA condensation. DNA content was assessed by measuring the UV fluorescence of propidium iodide-stained DNA. Apoptotic population analysis was performed using CellQuest software (Becton Dickinson, Franklin Lakes, NJ, USA).

\section{Isolation of RNA and quantitative real-time reverse transcriptase-PCR}

Total RNA was isolated from each cell culture flask (both untreated fibroblasts and those treated with DEB or radiation) using the TRI reagent (Ambion, Inc.) in accordance with the manufacturer's instructions. The quantity purified mRNA was assessed with a NanoDrop ${ }^{\circledR}$ ND-1000 
spectrophotometer (Thermo Scientific, Wilmington, Delaware). Structural RNA integrity was confirmed by formaldehyde gel electrophoresis. One microgram of RNA was treated with DNAse I (Fermentas, Lithuania) and reverse transcription was performed using a first strand cDNA synthesis kit with oligo-dT18 primers (Fermentas, Lithuania) according to the manufacturer's instructions. Mock reactions lacking reverse transcriptase (RT) were performed during the cDNA synthesis step to exclude genomic contamination. Real-time PCR was performed in duplicate in an ABI Real-time 7500 system (ABI, Foster City, CA). Detection of TRF-1 and TRF-2 gene expression was accomplished with $0.2 \mu \mathrm{mol} / \mathrm{L}$ of each primer and $0.1 \mu \mathrm{mol} / \mathrm{L}$ of probe in TaqMan ${ }^{\circledR}$ gene expression master mix in a total volume of $25 \mu \mathrm{L}$. The sequences of the primers and probes are as follows: TRF1, forward primer 5'-CCACATGATGG AGAAAATTAAGAGTTAT-3', reverse primer 5'-TGCC GCTGCCTTCA TTAGA-3, probe 5'-FAM-TTATGTG CTAAGTGAAAAATCATCAACCT-TAMRA-3' [52]. The results from the RT-PCR assays were validated by amplifying the serial dilutions of a sample by 10 -fold, and the slopes of amplification curves were calculated. Detection of internal reference $18 \mathrm{~s}$ rRNA was performed with predeveloped $\operatorname{TaqMan}^{\circledR}$ Gene Expression Assays ID Hs99999901_s1 (ABI, Foster City, CA), although the glyceraldehyde-3- phosphate dehydrogenase (GAPDH, Hs99999905_m1) and cyclophilin A (CYCA, Hs999999 04_m1) were run on all the samples as two additional endogenous controls. Differences in mRNA expression, according to genotypes of the investigated polymorphisms, were tested with REST 09 software (Corbett Life Science, http://rest.gene-quantification.info) [53].

\section{Statistics}

The data were analyzed using statistical software package 7.0. Statistical analyses were performed via Student's $t$ test. Correlations between investigated parameters were tested with linear regression analysis. A P value less than 0.05 was considered statistically significant. Differences in mRNA expression between groups were tested by pairwise randomization and boot strapping with the relative expression software tool, REST 09 (Corbett Life Science) [53].

\section{Competing interests}

The authors declare that they have no competing interests.

\footnotetext{
Authors' contribution

GJ and PS designed the study. IJ, AL, SP, MO, JP, MZ and AS carried out experiments. DV and MG-S provided clinical material and helped with the analysis. JS, PS and GJ supervised experimental work in their laboratories. GJ and PS wrote the manuscript. All authors approved the final version of the manuscript.
}

\section{Acknowledgement}

This work was supported by the Ministry of Education and Science of the Republic of Serbia (Project No.173046).

\section{Author details}

${ }^{1}$ Vinca Institute of Nuclear Sciences, University of Belgrade, Belgrade, Serbia. ${ }^{2}$ Hospital of Gynecology and obstetrics "Narodni front", Belgrade, Serbia. ${ }^{3}$ School of Medicine, University of Belgrade, Belgrade, Serbia. ${ }^{4}$ Mother and Child Health Care Institute of Serbia "Dr Vukan Cupic", Belgrade, Serbia. ${ }^{5}$ Faculty of Biology, University of Belgrade, Belgrade, Serbia. ${ }^{6}$ Brunel University, West London, United Kingdom. ${ }^{7}$ Department of Genetics and Microbiology, Universitat Autònoma de Barcelona, and Center for Biomedical Network Research on Rare Diseases (CIBERER), Bellaterra, Barcelona, Spain.

Received: 23 July 2012 Accepted: 9 September 2012

Published: 14 September 2012

\section{References}

1. Greider CW, Blackburn EH: Identification of a specific telomere terminal transferase activity in tetrahymena extracts. Cell 1985, 43:405-413.

2. de Lange $T$ : Shelterin: the protein complex that shapes and safeguards human telomeres. Genes Dev 2005, 19:2100-2110.

3. Smogorzewska A, van Steensel B, Bianchi A, Oelmann S, Schaefer MR, Schnapp G, de Lange T: Control of human telomere length by TRF1 and TRF2. Mol Cell Biol 2000, 20:1659-1668.

4. van Steensel B, Smogorzewska A, de Lange T: TRF2 protects human telomeres from end-to-end fusions. Cell 1998, 92:401-413.

5. Sfeir A, Kosiyatrakul ST, Hockemeyer D, MacRae SL, Karlseder J, Schildkraut $C L$, de Lange $T$ : Mammalian telomeres resemble fragile sites and require TRF1 for efficient replication. Cell 2009, 138:90-103.

6. Celli GB, de Lange T: DNA processing is not required for ATM-mediated telomere damage response after TRF2 deletion. Nat Cell Biol 2005, 7:712-718.

7. Celli GB, Denchi EL, de Lange T: Ku70 stimulates fusion of dysfunctional telomeres yet protects chromosome ends from homologous recombination. Nat Cell Biol 2006, 8:885-890.

8. Palm W, Hockemeyer D, Kibe T, de Lange T: Functional dissection of human and mouse POT1 proteins. Mol Cell Biol 2009, 29:471-482

9. Bakkenist CJ, Kastan MB: Initiating cellular stress responses. Cell 2004, 118:9-17.

10. d'Adda di Fagagna F, Reaper PM, Clay-Farrace L, Fiegler H, Carr P, Von Zglinicki T, Saretzki G, Carter NP, Jackson SP: A DNA damage checkpoint response in telomere-initiated senescence. Nature 2003, 426:194-198.

11. Takai H, Smogorzewska A, de Lange T: DNA damage foci at dysfunctional telomeres. Curr Biol 2003, 13:1549-1556.

12. Blasco MA: Telomeres and human disease: ageing, cancer and beyond. Nat Rev Genet 2005, 6:611-622.

13. Ball SE, Gibson FM, Rizzo S, Tooze JA, Marsh JC, Gordon-Smith EC: Progressive telomere shortening in aplastic anemia. Blood 1998, 91:3582-3592.

14. Callen E, Samper E, Ramirez MJ, Creus A, Marcos R, Ortega JJ, Olive T, Badell I, Blasco MA, Surralles J: Breaks at telomeres and TRF2-independent end fusions in fanconi anemia. Hum Mol Genet 2002, 11:439-444.

15. Taniguchi T, D'Andrea AD: Molecular pathogenesis of fanconi anemia: recent progress. Blood 2006, 107:4223-4233.

16. Dokal I, Vulliamy T: Inherited bone marrow failure syndromes. Haematologica 2010, 95:1236-1240.

17. Li X, Leteurtre F, Rocha V, Guardiola P, Berger R, Daniel MT, Noguera MH, Maarek O, Roux GL, de la Salmoniere $P$, et al: Abnormal telomere metabolism in fanconi's anaemia correlates with genomic instability and the probability of developing severe aplastic anaemia. Br J Haematol 2003, 120:836-845

18. Leskovac A, Vujic D, Guc-Scekic M, Petrovic S, Joksic I, Slijepcevic P, Joksic G: Fanconi anemia is characterized by delayed repair kinetics of DNA double-strand breaks. Tohoku J Exp Med 2010, 221:69-76.

19. Kalb R, Neveling K, Hoehn H, Schneider H, Linka Y, Batish SD, Hunt C, Berwick M, Callen E, Surralles J, et al: Hypomorphic mutations in the gene encoding a key fanconi anemia protein, FANCD2, sustain a significant group of FA-D2 patients with severe phenotype. Am J Hum Genet 2007, 80:895-910.

20. Leteurtre F, Li X, Guardiola P, Le Roux G, Sergere JC, Richard P, Carosella ED, Gluckman E: Accelerated telomere shortening and telomerase activation in fanconi's anaemia. Br J Haematol 1999, 105:883-893.

21. Hemann MT, Strong MA, Hao L-Y, Greider CW: The shortest telomere, not average telomere length, is critical for cell viability and chromosome stability. Cell 2001, 107:67-77. 
22. Blanco R, Munoz P, Flores JM, Klatt P, Blasco MA: Telomerase abrogation dramatically accelerates TRF2-induced epithelial carcinogenesis. Genes Dev 2007, 21:206-220.

23. Okamoto K, Iwano T, Tachibana M, Shinkai Y: Distinct roles of TRF1 in the regulation of telomere structure and lengthening. J Biol Chem 2008, 283:23981-23988.

24. Philippe C, Coullin P, Bernheim A: Double telomeric signals on single chromatids revealed by fish and prins. Ann Genet 1999, 42:202-209.

25. Undarmaa B, Kodama S, Suzuki K, Niwa O, Watanabe M: X-ray-induced telomeric instability in Atm-deficient mouse cells. Biochem Biophys Res Commun 2004, 315:51-58.

26. van Overbeek $M$, de Lange T: Apollo, an artemis-related nuclease, interacts with TRF2 and protects human telomeres in S phase. Curr Biol 2006, 16:1295-1302.

27. van Steensel B, de Lange T: Control of telomere length by the human telomeric protein TRF1. Nature 1997, 385:740-743.

28. Aragona M, Maisano R, Panetta S, Giudice A, Morelli M, La Torre I, La Torre F: Telomere length maintenance in aging and carcinogenesis. Int J Oncol 2000, 17:981-989.

29. Kishi S, Wulf G, Nakamura M, Lu KP: Telomeric protein Pin2/TRF1 induces mitotic entry and apoptosis in cells with short telomeres and is downregulated in human breast tumors. Oncogene 2001, 20:1497-1508.

30. Lyakhovich A, Ramirez MJ, Castellanos A, Castella M, Simons AM, Parvin JD, Surralles J: Fanconi anemia protein FANCD2 inhibits TRF1 polyADP-

ribosylation through tankyrase1-dependent manner. Genome Integr 2011, 2:4.

31. Polanska E, Dobsakova Z, Dvorackova M, Fajkus J, Stros M: HMGB1 gene knockout in mouse embryonic fibroblasts results in reduced telomerase activity and telomere dysfunction. Chromosoma 2012, 121:419-431.

32. Riethman H: Human telomere structure and biology. Annu Rev Genomics Hum Genet 2008, 9:1-19.

33. Schoeftner S, Blasco MA: Developmentally regulated transcription of mammalian telomeres by DNA-dependent RNA polymerase II. Nat Cell Biol 2008, 10:228-236.

34. Kaul Z, Cesare AJ, Huschtscha LI, Neumann AA, Reddel RR: Five dysfunctional telomeres predict onset of senescence in human cells. EMBO Rep 2012, 13:52-59.

35. Cabuy E, Newton C, Joksic G, Woodbine L, Koller B, Jeggo PA, Slijepcevic P. Accelerated telomere shortening and telomere abnormalities in radiosensitive cell lines. Radiat Res 2005, 164:53-62.

36. Slijepcevic $P$ : Is there a link between telomere maintenance and radio sensitivity? Radiat Res 2004, 161:82-86.

37. Thyagarajan B, Campbell C: Elevated homologous recombination activity in fanconi anemia fibroblasts. J Biol Chem 1997, 272:23328-23333.

38. Rouet P, Smih F, Jasin M: Expression of a site-specific endonuclease stimulates homologous recombination in mammalian cells. Proc Natl Acad Sci USA 1994, 91:6064-6068.

39. Yamamoto K, Hirano S, Ishiai M, Morishima K, Kitao H, Namikoshi K, Kimura M, Matsushita N, Arakawa H, Buerstedde JM, et al: Fanconi anemia protein FANCD2 promotes immunoglobulin gene conversion and DNA repair through a mechanism related to homologous recombination. $\mathrm{Mol} \mathrm{Cell}$ Biol 2005, 25:34-43.

40. Nakanishi $K$, Yang YG, Pierce AJ, Taniguchi T, Digweed M, D'Andrea AD, Wang $Z Q$, Jasin M: Human fanconi anemia monoubiquitination pathway promotes homologous DNA repair. Proc Natl Acad Sci USA 2005, 102:1110-1115.

41. Smogorzewska A, Matsuoka S, Vinciguerra P, McDonald ER 3rd, Hurov KE, Luo J, Ballif BA, Gygi SP, Hofmann K, D'Andrea AD, Elledge SJ: Identification of the $\mathrm{FANCI}$ protein, a monoubiquitinated $\mathrm{FANCD} 2$ paralog required for DNA repair. Cell 2007, 129:289-301.

42. Broccoli D, Smogorzewska A, Chong L, de Lange T: Human telomeres contain two distinct Myb-related proteins, TRF1 and TRF2. Nat Genet 1997, 17:231-235.

43. Morrish TA, Greider CW: Short telomeres initiate telomere recombination in primary and tumor cells. PLoS Genet 2009, 5:e1000357.

44. Fan Q, Zhang F, Barrett B, Ren K, Andreassen PR: A role for monoubiquitinated FANCD2 at telomeres in ALT cells. Nucleic Acids Res 2009, 37:1740-1754.

45. Cesare AJ, Kaul Z, Cohen SB, Napier CE, Pickett HA, Neumann AA, Reddel RR: Spontaneous occurrence of telomeric DNA damage response in the absence of chromosome fusions. Nat Struct Mol Biol 2009, 16:1244-1251.
46. Auerbach AD: Diagnosis of fanconi anemia by diepoxybutane analysis. In Current Protocols in Human Genetics. Edited by Dracopoli NC, Haines JL, Korf BR, Moir DT, Morton CC, Seidman CE, Seidman JG, Smith DR. Hoboken, NJ: ohn Wiley \& Sons, Inc; 2003:8.7.1-8.7.15.

47. Casado AJ, Callen E, Jacome A, Rio P, Castella M, Lobitz S, Ferro T, Munoz A, Sevilla J, Cantalejo A, et al: A comprehensive strategy for the subtyping of patients with fanconi anaemia: conclusions from the spanish fanconi anemia research network. J Med Genet 2007, 44:241-249.

48. Castella M, Pujol R, Callen E, Trujillo JP, Casado JA, Gille H, Lach FP, Auerbach AD, Schindler D, Benitez J, et al: Origin, functional role, and clinical impact of fanconi anemia FANCA mutations. Blood 2011, 117:3759-3769.

49. Slijepcevic P: Telomere length measurement by Q-FISH. Methods Cell Sci 2001, 23:17-22.

50. Perry $P$, Wolff S: New Giemsa method for the differential staining of sister chromatids. Nature 1974, 251:156-158.

51. Bailey SM, Goodwin EH, Meyne J, Cornforth MN: CO-FISH reveals inversions associated with isochromosome formation. Mutagenesis 1996, 11:139-144.

52. Lin X, Gu J, Lu C, Spitz MR, Wu X: Expression of telomere-associated genes as prognostic markers for overall survival in patients with non-small cell lung cancer. Clin Cancer Res 2006, 12:5720-5725.

53. Pfaffl MW, Horgan GW, Dempfle L: Relative expression software tool (REST) for group-wise comparison and statistical analysis of relative expression results in real-time PCR. Nucleic Acids Res 2002, 30:e36.

doi:10.1186/2041-9414-3-6

Cite this article as: Joksic et al:: Dysfunctional telomeres in primary cells from Fanconi anemia FANCD2 patients. Genome Integrity 2012 3:6.

\section{Submit your next manuscript to BioMed Central and take full advantage of:}

- Convenient online submission

- Thorough peer review

- No space constraints or color figure charges

- Immediate publication on acceptance

- Inclusion in PubMed, CAS, Scopus and Google Scholar

- Research which is freely available for redistribution

Submit your manuscript at www.biomedcentral.com/submit
C) Biomed Central 